\title{
Pathological Count of Igg4-Positive Plasmacytes Suggests Extraophthalmic Involvement And Relapse In Patients With Igg4-Related Ophthalmic Disease: A Retrospective Study
}

\section{Yiqun Yuan}

Eye \& ENT Hospital of Fudan University

\section{Fengxi Meng}

Eye \& ENT Hospital of Fudan University

Hui Ren

Eye \& ENT Hospital of Fudan University

Han Yue

Eye \& ENT Hospital of Fudan University

Kang Xue

Eye \& ENT Hospital of Fudan University

Rui Zhang ( $\nabla$ zhangrui936@163.com )

Eye \& ENT Hospital of Fudan University

\section{Research Article}

Keywords: IgG4-related ophthalmic disease, pathological findings, serum IgG4 level, systemic involvement, relapse risk

Posted Date: January 31st, 2022

DOI: https://doi.org/10.21203/rs.3.rs-1301486/v1

License: (9) This work is licensed under a Creative Commons Attribution 4.0 International License. Read Full License

Version of Record: A version of this preprint was published at Arthritis Research \&amp; Therapy on April 1st, 2022. See the published version at https://doi.org/10.1186/s13075-022-02757-2. 


\section{Abstract}

Background: IgG4-related ophthalmic disease (IgG4ROD) is a phenotype of IgG4-related disease (IgG4RD) with ophthalmic involvement. The pathological IgG4+ plasmacyte count has only been used for diagnosis. We aimed to explore its possible clinical value in the management of IgG4ROD.

Methods: Fifty-five pathologically diagnosed IgG4ROD patients were included, and their clinical, pathological, serological, and radiological findings and treatment outcomes were reviewed and analyzed. The pathological IgG4+ plasmacyte counts in lesions from different anatomic sites were compared, and their association with serum IgG4 concentrations, systemic involvement and relapse risk was analyzed.

Results: The patients were divided into groups according to the anatomic site of their biopsied lesions, namely, the lacrimal gland, extraocular muscle and orbital soft tissue. No significant difference was found in the pathological IgG4+ plasma cell counts among these groups $(p=0.975)$. The pathological IgG4+ plasmacyte count positively correlated with the $\lg G 4$ concentration in peripheral blood $\left(R^{2}=0.5469\right.$, $p<0.001)$. The serum IgG4 concentration and the pathological infiltrating IgG4+ plasmacyte count were significantly higher in patients with extraophthalmic involvement ( $p<0.001$ and $p=0.005$, respectively). The areas under the receiver operating characteristic (ROC) curve (AUCs) of the serum IgG4 level and pathological IgG4+ plasmacyte count for identifying systemic involvement were $0.897(p<0.001)$ and $0.759(p=0.015)$, respectively. The patients with relapse had higher levels of serum IgG4, more germinal centers (GCs), and infiltrating IgG4+ plasmacytes in lesions. Multivariate Cox regression analysis revealed that a pathological IgG4+ plasmacyte count of $>150 /$ high-power field (HPF) and an elevated serum IgG4 level of $>500 \mathrm{mg} / \mathrm{dL}$ were risk factors for relapse after steroid treatment.

Conclusions: Lesions from different ophthalmic sites in IgG4ROD patients have similar counts of IgG4+ and IgG+ plasmacytes. The quantity of pathological IgG4+ plasmacytes corresponded to the serum IgG4 concentration in patients with IgG4ROD and could be meaningful in identifying systemic involvement and predicting subsequent relapse.

\section{Background}

IgG4-related ophthalmic disease (IgG4ROD) is a recently recognized condition characterized by tumefacient ophthalmic lesions infiltrated by IgG4-positive (IgG4+) plasmacytes [1]. The histopathological feature of dense IgG4+ plasmacyte infiltration is the most essential hallmark for diagnosing this condition [2, 3]. IgG4ROD shares similarities with other inflammatory diseases referred to as IgG4-related diseases (IgG4RDs). A significant proportion of patients with IgG4ROD have systemic disorders such as sialadenitis, thyroiditis, parotitis, and retroperitoneal fibrosis [4]. In addition, most IgG4ROD patients show elevated concentrations of IgG4 antibody in peripheral blood. Although the disease responds well to corticosteroids, nearly $50 \%$ of patients experience recurrence after steroid treatment [5]. 
Accumulating studies of IgG4RD have demonstrated the usefulness of serum IgG4 levels in evaluating disease severity and monitoring relapse [6-8]. The serum IgG4 concentration score is an important part of the IgG4RD responder index for identifying activity [9]. Autoimmune pancreatitis (AIP) patients with elevated serum IgG4 concentrations have been reported to have a higher incidence of severe symptoms and extrapancreatic lesions [10]. A prospective IgG4RD cohort study in the UK suggested that a serum lgG4 level of $\geq 280 \mathrm{mg} / \mathrm{dL}$ at diagnosis is useful for predicting multiorgan involvement and the risk of relapse [11]. The relationship between the numbers of IgG4+ plasma cells and serum IgG4 concentrations remains obscure, although several previous studies have noticed that the counts of IgG4+ plasma cells tend to be higher in patients with AIP and other non-IgG4-related diseases and higher IgG4 concentrations $[12,13]$. The count of IgG4+ plasmacytes infiltrating lesions has only been applied to diagnosis.

Before investigating the clinical value of pathological IgG4+ plasmacytes, we noticed that the numbers of IgG4+ cells infiltrating different lesions varied from organ to organ [14]. Quite different cutoff numbers of IgG4+ plasmacytes were applied to organ-specific IgG4RD diagnosis criteria. IgG4ROD describes IgG4RDs that occur in ophthalmic sites, including the lacrimal glands, trigeminal nerves, extraocular muscles and other orbital soft tissues [15]. The histogenesis and histologic characteristics vary in different ophthalmic sites. It has not been determined whether the counts of IgG4+ plasma cells in different ophthalmic sites are similar. In this study, we calculated the pathological counts of IgG4+ plasma cells and investigated their potential role in the management of IgG4ROD.

\section{Methods}

\section{Patients and tissue samples}

This study was a retrospective review. Consecutive patients with a diagnosis of IgG4ROD referred to the Eye and ENT hospital of Fudan University were selected between 2016 and 2020. No corticosteroid or immunosuppressive treatment was given in the 3 months prior to when tissue and blood samples were obtained. All the biopsy samples were excised from the major ophthalmic lesions. The biopsy sections of all the patients were reviewed, and a histopathological diagnosis of IgG4ROD was made by one experienced pathologist after ruling out all potential alternative diagnoses in accordance with the diagnostic criteria proposed in 2014.

\section{Histopathological data}

Formalin-fixed and paraffin-embedded sections were stained with hematoxylin and eosin (HE). Slides of immunohistochemical staining of IgG4 and IgG (or CD138) were reviewed. IgG4+ plasma cells were counted in 5 nonoverlapping high-power fields (HPFs) with the highest density of stained positive cells at $40^{\prime}$ magnification. The average count was recorded. The average number of Ig G+ plasma cells was also calculated in the fields where IgG4+ plasma cells were counted. Furthermore, HE-stained biopsy slides were morphologically screened for the presence of germinal centers (GCs), which was confirmed 
according to CD21 staining. The average number of GCs from 5 low-power fields (LPFs) with the densest concentration of lymphoid follicles at $5^{\prime}$ magnification was counted.

\section{Clinical data}

The electronic clinical records of all the patients were retrieved. All the patients underwent an extensive medical examination, and patients' symptoms, laterality, and lesion locations were recorded. All 55 patients underwent axial and coronal magnetic resonance imaging (MRI) or computed tomography (CT) scans of the orbit, head and neck before biopsy. Major ophthalmic lesions were classified by their anatomical location. Extraophthalmic lesions of the head and neck were determined by analyzing radiological images and performing a physical examination. Serum IgG4 concentration examinations were performed on peripheral blood from 31 patients at the time of diagnosis. The history of steroid treatment was recorded, and the treatment response and subsequent relapse after glucocorticoid regimens were reviewed. All the patients were followed up for more than 1 year.

\section{Statistical analysis}

The analyses of the pathological counts of IgG4+ and IgG+ plasmacytes and GCs among the three groups were conducted using one-way ANOVA and LSD tests. The logarithm of the serum IgG4 level was introduced in a parametric test for the concentration following the lognormal distribution in IgG4ROD. The difference in the histopathological and serological findings between the groups with and without extraophthalmic involvement was analyzed using the unpaired Student's $t$ test. Linear regression analysis was performed to analyze the association between histopathological factors and serum IgG4 levels. Receiver operating characteristic (ROC) curve analysis was used to calculate the area under the curve (AUC) to identify diagnostic values of the counts of IgG4+ plasmacytes and serum IgG4 levels for IgG4ROD. Univariate and multivariate Cox regression analyses (enter method) were performed to identify the potential clinicopathological risk factors for relapse and their hazard ratios (HRs). Variables with $p<0.1$ in the univariate Cox regression were included in the multivariate analysis. Kaplan-Meier survival curves and log-rank tests were performed to compare the effect of different grades of IgG4+ plasmacyte infiltration on IgG4ROD relapse. $p<0.05$ was considered statistically significant. All statistical analyses were performed with SPSS statistics v.25 and R-project 4.1.2.

\section{Results}

Fifty-five consecutive patients ( 30 men, 25 women; age range 15-72 years; mean age 54.5 years) were histopathologically diagnosed with IgG4ROD according to the diagnostic criteria. Fifty-one patients' IgG4 immunostaining sections demonstrated $>50$ positive cells per HPF; although the lgG4-expressing plasmacytes per HPF were less than 50 in the 4 remaining patients, they were diagnosed with IgG4ROD based on the criterion of an IgG4+/lgG+ ratio $>40 \%$. Bilateral lesions were found in $27(49.1 \%)$ patients, and the rest exhibited unilateral lesions. The locations of the lesions were as follows: lacrimal gland, 43 (78.2\%); extraocular muscle, 19 (34.5\%); intraconal orbital soft tissue, 15 (27.3\%); extraconal orbital soft tissue, 23 (41.8\%); infraorbital nerve, 8 (14.5\%); and conjunctiva, 1 (1.8\%). 


\section{Pathological findings in IgG4ROD lesions}

All the biopsy samples were excised from the major ophthalmic lesions: $38(69.1 \%)$ from the lacrimal gland, 6 (10.9\%) from the extraocular muscle, 1 (1.8\%) from the conjunctiva, and 10 (18.2\%) from orbital soft tissues (extraconal and intraconal). All the IgG4ROD samples except one sample obtained from the conjunctiva were divided into three groups based on the anatomic location of the biopsy: the lacrimal gland group, the extraocular muscle group, and the orbital soft tissue group. Histopathologic examination of all the samples showed marked lymphocyte and plasmacyte infiltration.

\section{GCs in IgG4ROD lesions}

GCs were observed in all the patients and were further confirmed by reviewing immunostaining for the follicular dendritic cell marker CD21 (Figure 1a and b). The numbers of GCs in different lesions for the lacrimal gland, extraocular muscle, and orbital soft tissue groups were $6.47 \pm 2.45,3.03 \pm 2.57$, and $4.68 \pm 3.42$, respectively. A significant difference was found in the multiple comparison test among the three groups ( $p=0.008$, the lacrimal gland group vs. the extraocular muscle group, $p=0.005$, Figure 2a).

\section{IgG4+ plasma cells in IgG4ROD lesions}

In our study, IgG4+ plasma cells were detected in the regions surrounding lymphoid follicles and were usually associated with fibrosis in the majority of our samples (Figure 1c). In some cases, however, the IgG4+ plasma cells were mainly confined to GCs (Figure 1d). The IgG4+ plasmacyte counts in the lacrimal gland, extraocular muscle, and orbital soft tissue groups were 126.6 $557.2,128.1 \pm 59.4$, and $122.2 \pm 72.6$, respectively, and were not significantly different $(p=0.975$, Figure $2 b)$. The IgG+ plasmacyte counts were also calculated, and those for the lacrimal gland, extraocular muscle, and orbital soft tissue groups were $190.8 \pm 78.0,164.6 \pm 76.3$, and $182.7 \pm 58.0$, respectively, and were not significantly different $(p=0.734$, Figure $2 c)$. In addition, the $\operatorname{lgG} 4+/ \operatorname{lgG}+$ ratios in the lacrimal gland, extraocular muscle and orbital soft tissue groups were $69.1 \pm 21.4 \%, 77.0 \pm 16.7 \%$, and $61.0 \pm 25.3 \%$, respectively, and were not significantly different $(p=0.487$, Figure $2 \mathrm{~d})$.

\section{Relationship between GC formation and plasmacyte infiltration}

The pathological IgG4+ plasmacyte counts were positively correlated with the number of GCs in ophthalmic lesions $\left(p=0.002<0.05, \mathrm{R}^{2}=0.175\right.$, Figure $\left.2 \mathrm{e}\right)$. The IgG4+/lgG+ ratios were also slightly positively correlated with the GC counts in LPFs $\left(p=0.012, \mathrm{R}^{2}=0.1003\right.$, Figure $\left.2 \mathrm{f}\right)$. There was no correlation between the number of GCs and the pathological count of IgG+ plasmacytes $\left(p=0.167, \mathrm{R}^{2}=0.0299\right.$, figure not shown).

\section{Systemic conditions in patients with IgG4ROD}

Serum IgG4 level in IgG4ROD patients 
The serum IgG4 concentration was greater than $135 \mathrm{mg} / \mathrm{dL}$ in 24 (77.4\%) of the 31 lgG4ROD patients whose peripheral blood had been tested at diagnosis. The serum IgG4 levels are indicated with medians and quartiles, and the median concentration was $376 \mathrm{mg} / \mathrm{dL}$ (interquartile range 139 to $866 \mathrm{mg} / \mathrm{dL}$ ). As shown, the frequency of the serum IgG4 level in the IgG4ROD patients displayed a highly skewed distribution (Figure 3a). The logarithm of the serum IgG4 concentration exhibited a normal distribution $(p=0.533$, Figure $3 b)$.

\section{Relationship between serum IgG4 level and plasmacyte infiltration}

The logarithm of the serum IgG4 level was included in the analysis of the association between the serum IgG4 concentration and the number of infiltrating plasmacytes. As shown, the counts of IgG4+ plasma cells and the logarithm of the serum IgG4+ level showed a strong and significantly positive correlation ( $p<0.001, \mathrm{R}^{2}=0.5469$, Figure $3 \mathrm{c}$ ). The $\lg \mathrm{G} 4+/ \mathrm{lg} \mathrm{G}+$ ratio and $\mathrm{GC}$ count were also positively correlated with the $\operatorname{lgG} 4$ concentration in the peripheral blood with a rather lower coefficient of determination $(p<0.05$, Figure $3 \mathrm{~d}$ and $\mathrm{e})$.

\section{Extraophthalmic lesions of the head and neck}

Sixteen $(29.1 \%)$ of the 55 histopathologically diagnosed patients with IgG4ROD had extraophthalmic lesions of the head and neck. These lesions were observed on the pituitary gland in 2 patients, the parotid gland in 5 patients, the submandibular gland in 7 patients, the lymph nodes in 2 patients, the thyroid gland in 1 patient, the pterygopalatine fossa in 8 patients, the infratemporal fossa in 6 patients, and the cheek skin in 2 patients. Compared with those without multiorgan involvement in the head and neck, the patients with multiorgan involvement exhibited significantly higher serum IgG4 concentrations and pathological counts of infiltrating IgG4+ plasmacytes. These patients also presented a higher ratio of pathological IgG4+ plasmacytes to IgG+ plasmacytes and a higher rate of bilaterality (Table 1).

Table 1. Comparison of the clinicopathological findings in patients with and without extraophthalmic lesions of the head and neck 


\begin{tabular}{|c|c|c|c|}
\hline \multirow[t]{2}{*}{ Clinicopathological factor } & \multicolumn{2}{|c|}{ Extraophthalmic lesions of head and neck } & \multirow[t]{2}{*}{$p$ Value } \\
\hline & with $(n=16,29.1 \%)$ & without $(n=39,70.9 \%)$ & \\
\hline Age of disease onset (years) & $54.1 \pm 10.7$ & $51.8 \pm 12.5$ & 0.298 \\
\hline Sex (male, \%) & 51.3 & 62.5 & 0.448 \\
\hline Bilaterality (\%) & 75 & 38.5 & $0.014^{*}$ \\
\hline Lacrimal gland involvement (\%) & 87.5 & 74.4 & 0.284 \\
\hline In(serum IgG4 level) & $6.8 \pm 0.7$ & $5.2 \pm 1.2$ & $<0.001 *$ \\
\hline GC (LPF) & $6.7 \pm 2.5$ & $5.3 \pm 2.9$ & 0.099 \\
\hline Pathological IgG4+ plasmacyte (HPF) & $161.9 \pm 49.5$ & $113.1 \pm 58.1$ & $0.005^{*}$ \\
\hline Pathological IgG+ plasmacyte (HPF) & $201.2 \pm 64.0$ & $185.4 \pm 77.9$ & 0.476 \\
\hline Pathological lgG4+/lgG+ ratio (\%) & $82.8 \pm 11.5$ & $63.6 \pm 22.5$ & $0.002^{*}$ \\
\hline
\end{tabular}

GC germinal center, ${ }^{*} p<0.05$

The performance of the serum IgG4 level and the pathological count of IgG4+ plasmacytes for predicting systemic involvement in patients with IgG4ROD was evaluated by ROC curve analysis. The AUCs of the serum IgG4 level, pathological IgG4+ plasmacyte count and IgG4+/lgG+ ratio were 0.897 (95\% Cl: $0.777-$ $1.000, p<0.001), 0.759$ (95\% Cl: $0.589-0.928, p=0.015)$, and 0.669 (95\% Cl: $0.476-0.862, p=0.114$, Figure $3 f)$, respectively. The AUC using the serum IgG4 level $(=0.934)$ and the pathological count of IgG4+ plasmacytes $(=0.745)$ was moderately accurate for predicting systemic involvement in patients with IgG4ROD because both AUC measures fell between 0.7 and 0.9 . The largest Youden index of 0.735 [(sensitivity-[1-specificity]=0.735)] suggested a cutoff value for the serum IgG4 level of $478.5 \mathrm{mg} / \mathrm{dL}$. The sensitivity and specificity were 0.846 and 0.889 , respectively. Regarding the pathological lgG4+ plasmacyte count, the largest Youden index (0.457) suggested a cutoff value of 130/HPF, which resulted in a sensitivity of 0.846 and specificity of 0.611 .

\section{Treatment and IgG4ROD relapse}

\section{Treatment and relapse}

Forty-six of the 55 patients received oral steroid regimens as their initial postoperative treatment. Oral glucocorticoids were given at an initial dose of $0.5-0.6 \mathrm{mg} / \mathrm{kg} /$ day for 4 weeks and were then tapered over the following 6-8 weeks. One patient who underwent steroid withdrawal during the initial glucocorticoid treatment was excluded from this part of the study. Forty-one (91.1\%) of the 45 patients who took glucocorticoids responded well, and clinical remission was not achieved in the remaining patients. Twenty-four (58.5\%) of the 41 patients in whom remission was achieved suffered recurrence during the follow-up period. Thirteen (54.2\%) of the 24 relapses occurred within 6 months 
following steroid treatment. As shown in Table 2, the patients with relapse had a higher baseline level of serum IgG4 and more infiltrating plasmacytes in lesions. More GCs were also be observed in their samples.

Table 2. Comparison of the clinicopathological findings in IgG4ROD patients with and without relapse after steroid treatment

\begin{tabular}{|llll|}
\hline Clinicopathological factor & \multicolumn{2}{c|}{ Relapse after steroid treatment } & \multirow{2}{*}{$p$ Value } \\
\cline { 2 - 3 } & with (n=24, 58.5\%) & without (n=17, 41.5\%) & \\
\hline Age of disease onset (years) & $54.7 \pm 8.5$ & $48.8 \pm 16.3$ & 0.135 \\
\hline Sex (male, \%) & 50.0 & 47.1 & 0.853 \\
\hline Bilaterality (\%) & 62.5 & 47.1 & 0.326 \\
\hline Lacrimal gland involvement (\%) & 79.2 & 76.5 & 0.837 \\
\hline In(serum IgG4 level) & $6.4 \pm 0.99$ & $5.0 \pm 1.26$ & $\mathbf{0 . 0 0 2 *}$ \\
\hline GC (LPF) & $7.2 \pm 2.7$ & $4.0 \pm 2.4$ & $<0.001^{*}$ \\
\hline Pathological IgG4+ plasmacyte (HPF) & $155.7 \pm 51.2$ & $94.6 \pm 49.0$ & $<0.00$ * $^{*}$ \\
\hline Pathological IgG+ plasmacyte (HPF) & $199.42 \pm 58.5$ & $167.0 \pm 74.4$ & 0.127 \\
\hline Pathological IgG4+/lgG+ ratio (\%) & $80.4 \pm 16.2$ & $59.9 \pm 21.8$ & $\mathbf{0 . 0 0 1 *}$ \\
\hline Systemic involvement (head and neck, \%) & 45.8 & 23.5 & 0.144 \\
\hline
\end{tabular}

GC germinal center, ${ }^{\star} p<0.05$

Factors associated with relapse

Through univariate Cox regression analysis, we identified that systemic involvement (head and neck), pathological GC and IgG4+ plasmacyte counts, and the level of serum IgG4 were associated with relapse (Table 3). Multivariate Cox regression analysis revealed that infiltrating IgG4+ plasmacytes $>150 / \mathrm{HPF}$ (HR 7.63, 95\% Cl 1.27-45.97, $p=0.027$ ) and elevated serum IgG4 levels $>500$ mg/dL (HR 5.06, 95\% Cl 1.1522.35, $p=0.032$ ) were risk factors for relapse (Figure 4a). Kaplan-Meier curves of the cumulative relapse rate of IgG4ROD patients based on the numbers of pathological IgG4+ plasmacytes are plotted in Figure $3 \mathrm{~b}$. The log-rank test proved that there was a significant difference between the cumulative relapse curves of the IgG4ROD patients with different degrees of IgG4+ plasmacyte infiltration. The patients with $>150 /$ HPF pathological IgG4+ plasmacytes tended to have a higher cumulative relapse rate than those with fewer IgG4+ plasmacytes in lesions.

Table 3. Univariate Cox regression analysis for IgG4ROD relapse 


\begin{tabular}{|c|c|c|c|}
\hline Clinicopathological factor & Number $[\% \square$ & $\mathrm{HR}(95 \% \mathrm{Cl})$ & $p$ Value \\
\hline Age of disease onset (years) & $41(100)$ & $1.017(0.985-1.050)$ & 0.289 \\
\hline \multicolumn{4}{|l|}{ Sex } \\
\hline male & $20(48.8)$ & 1 & Ref \\
\hline female & $21(51.2)$ & $0.767(0.344-1.709)$ & 0.516 \\
\hline \multicolumn{4}{|l|}{ Bilateral } \\
\hline yes & $23(56.1)$ & 1 & Ref \\
\hline no & $18(43.9)$ & $0.567(0.247-1.301)$ & 0.199 \\
\hline \multicolumn{4}{|l|}{ Lacrimal gland involvement } \\
\hline yes & $32(78.0)$ & 1 & Ref \\
\hline no & $9(22.0)$ & $0.956(0.584-1.567)$ & 0.859 \\
\hline \multicolumn{4}{|c|}{ Systemic involvement (head and neck) } \\
\hline yes & $15(36.6)$ & 1 & Ref \\
\hline no & $26(63.4)$ & $0.711(0.476-1.064)$ & $0.097^{\#}$ \\
\hline \multicolumn{4}{|l|}{ GC (/LPF) } \\
\hline$<6$ & $19(46.3)$ & 1 & Ref \\
\hline$>=6$ & $22(53.7)$ & $4.073(1.603-10.350)$ & $0.003^{\#}$ \\
\hline \multicolumn{4}{|c|}{ Pathological IgG4+ plasmacyte (/HPF) } \\
\hline$<100$ & $14(34.1)$ & 1 & Ref \\
\hline $100-150$ & $11(26.9)$ & $4.697(1.242-17.758)$ & $0.023^{\#}$ \\
\hline$>150$ & $16(39.0)$ & $8.701(2.394-31.620)$ & $0.001^{\#}$ \\
\hline \multicolumn{4}{|l|}{ Serum IgG4 level (mg/dL) } \\
\hline$<500$ & $16(39.0)$ & 1 & Ref \\
\hline$>=500$ & $13(31.7)$ & $4.055(1.541-10.676)$ & $0.005^{\#}$ \\
\hline
\end{tabular}

HR hazard ratio, Cl confidence interval, Ref reference, GC germinal center, ${ }^{*} p<0.1$

\section{Discussion}


Histopathological features and immunohistochemical staining results provide strong supportive evidence for the diagnosis of IgG4RD. Although all conditions within the spectrum of IgG4RD are associated with lesions rich in IgG4+ plasma cells, a certain variability in the count exists across organs. Generally, lesions in exocrine glands such as the lacrimal glands tend to have strikingly more lgG4+ plasmacytes than organs such as the pancreas and retroperitoneum, with a predominance of fibrosis. We summarized the samples from lesions in the lacrimal glands, extraocular muscles, and orbital soft tissues. Lesions in the extraocular muscles and orbital soft tissues shared similar pathological features with those in the lacrimal glands [16]. Several ophthalmic lesions had no significant difference in the quantitative findings of IgG4 and IgG staining or their ratio. This result suggested that there was no significant variability in the cutoff number of IgG4+ and IgG+ plasmacytes across lesions in the different anatomical sites in IgG4ROD patients.

Even though an elevated serum IgG4 concentration is not sufficient to diagnose IgG4RD, its clinical interpretation has been established. There is convincing evidence that the serum level of $\operatorname{lgG} 4$ in patients with multiple organ involvement is higher and correlated with disease activity [17]. In our study, the IgG4 concentration distribution skewed to the left. To improve the efficiency of the analysis, we performed a parameter test after certifying that the baseline serum IgG4 level in the IgG4ROD patients showed a logarithmic normal distribution. The serum IgG4 level of patients with a pathological diagnosis of IgG4RD from previous studies also followed a logarithmic normal distribution ( $p=0.363, p=0.940$ and $p=0.236$, respectively) $[8,16,18]$. Performing a parameter test makes the positive correlation between the quantity of pathological factors and the serum IgG4 concentration more convincing. A higher coefficient of determination was found for IgG4 staining than for IgG staining. It is possible that the counts of IgG+ plasma cells in fields were much more likely to reach saturation, which diluted their relevance to serum IgG4 levels. Similar results were found for other IgG+-related factors. Our study was the first to analyze the relationship between IgG4 levels in serum and IgG4+ plasmacytes in lesions. Considering the clinical application of serum IgG4 levels, this result provided evidence for the potential application of IgG4+ plasmacyte counts in the management of IgG4ROD.

We investigated the potential value of the count of IgG4+ plasmacytes to identify systemic involvement. Because extraorbital imaging was not performed in most patients, we chose extraophthalmic lesions of the head and neck to represent systemic conditions. It has been reported that the head and neck region is the second most common site for IgG4RD after the pancreas [19]. The patients without any extraophthalmic symptoms or lesions of the head and neck were regarded as having no systemic involvement. Lesions in the pterygopalatine fossa and infratemporal fossa were regarded as extraophthalmic manifestations because we noticed that the two mentioned lesions in most cases were isolated $[15,20]$. Our results showed that the serum IgG4 level and the rate of bilaterality were higher in patients with systemic involvement. This result was consistent with that of a previous report [21]. More IgG4+ plasmacytes and a higher lgG4+/lgG+ ratio were also observed in these patient sections. The AUC using the serum IgG4 level was larger than that using the pathological IgG4+ plasmacyte count. For the identification of systemic lesions, a serum IgG4 cutoff value of $478.5 \mathrm{mg} / \mathrm{dL}$ had the same sensitivity of 0.846 and a higher specificity of 0.889 than a cutoff value of 130/HPF of pathological IgG4+ 
plasmacytes with a specificity of 0.661 . This implies that compared with the serum IgG4 level, the quantity of pathological IgG4+ plasmacytes may have a relatively weaker efficiency for indicating systemic involvement in IgG4ROD patients. This result may be able to apply to other subtypes of IgG4RD with similarly numerous infiltrating IgG4+ plasmacytes.

Apart from the function in identifying systemic involvement, we also investigated the role of IgG4+ plasmacyte-related pathological findings in predicting relapse. Consistent with a previous study, the patients with elevated pretreatment levels of serum IgG4 were more likely to experience relapse after steroid treatment. The patients with relapse also tended to have more infiltrating IgG4+ plasmacytes and more GCs in lesions. Multivariate Cox regression analysis indicated that a $>150 /$ HPF IgG4+ plasmacyte count and a $>500 \mathrm{mg} / \mathrm{dL}$ serum IgG4 level were risk factors for relapse. The practical value and quantity of pathological IgG4+ plasmacytes corresponded to the serum IgG4 concentration in patients with IgG4ROD.

The GC, the discrete lymphoid structure where high affinity B cells are selected and differentiate into plasma cells, is responsible for the generation of high-affinity IgG antibodies [22]. Compared to lesions in other ophthalmic sites, lesions in the lacrimal glands had more GCs. We found a positive correlation between the counts of GCs and IgG4+ plasmacytes with a low coefficient of determination. The positive correlation of the number of GCs with serum IgG4 levels and IgG4+ plasmacyte counts suggested that the development of IgG4 antibodies and IgG4+ plasmacytes may be associated with GCs. More GCs were observed in patients with relapse, but it was not the key risk factor for relapse in our study. This result indicates its potential value in clinical practice.

Taken together, this study demonstrated that the serum IgG4 level, which has been reported in previous studies, is of practical value in identifying systemic involvement and predicting relapse. The pathological IgG4+ plasmacyte count reflects the inflammation in localized lesions and is associated with local recurrence. It can also be used to identify systemic conditions with a lower specificity compared to that for the serum IgG4 level. The serum IgG4 level can be influenced by previous treatment, but baseline counts of pathological IgG4+ plasmacytes are always stable. This objective advantage of the pathological lgG4+ plasmacyte count over the serum IgG4 level lends itself to the management of IgG4ROD.

There were some limitations in this study. First, the subjects were limited to patients who were diagnosed pathologically. Second, systemic imaging examinations were not performed to evaluate extraophthalmic involvement, and the serum IgG4 concentration was tested only in some patients. Due to the lack of sufficient information on systemic conditions, our results on systemic involvement might not be accurate. Third, some patients were referred to us after receiving oral or intravenous steroid treatment and experiencing recurrence elsewhere. This could influence the analysis of response to steroids and relapse.

In conclusion, our results suggest that various lesions from different ophthalmic sites provide similar quantitative results regarding IgG4 and IgG staining in patients with IgG4ROD. The number and ratio of IgG4+ plasmacytes are positively correlated with the serum IgG4 level. Both higher serum IgG4 levels and 
more infiltrating IgG4+ plasmacytes are associated with systemic lesions and relapse. The pathological IgG4+ plasmacyte count may be of practical value in the management of IgG4ROD.

\section{List Of Abbreviations}

IgG: Immunoglobulin G; IgG4: Immunoglobulin G4; IgG4ROD: IgG4-related ophthalmic disease; IgG4RD: IgG4-related disease; GC: Germinal center; HPF: High-power field; LPF: Low-power field; LG: Lacrimal gland; EOM: Extraocular muscle; CT: Computed tomography; MRI: Magnetic resonance imaging; HR: Hazard ratio; SD: Standard; Cl: Confidence interval; Ref: Reference; ROC: Receiver operating characteristic; AUC: Area under the curve

\section{Declarations}

\section{Ethics approval and consent to participate}

The Medical Ethics Committee of the Eye \& ENT Hospital of Fudan University approved this study.

\section{Consent for publication}

Not applicable.

\section{Availability of data and materials}

The datasets used and/or analyzed during the current study are available from the corresponding author on reasonable request.

\section{Competing interests}

The authors declare that they have no competing interests.

\section{Funding}

This study was supported by grants from the Shanghai Science and Technology Planning Project [20Y11911200].

\section{Authors' contributions}

YQY and RZ conceived and designed the research; HR, HY and KX collected the data; YQY and FXM analyzed the data and wrote the manuscript; RZ revised the manuscript. The authors read and approved the final manuscript.

\section{Acknowledgements}

We want to thank Dr. Yingwen Bi for the pathological work. 


\section{References}

1. Kamisawa T, Zen Y, Pillai S, Stone JH. IgG4-related disease. Lancet. 2015;385:1460-71.

2. Goto H, Takahira M, Azumi A. Diagnostic criteria for IgG4-related ophthalmic disease. Jpn J Ophthalmol. 2015;59:1-7.

3. Wallace ZS, Naden RP, Chari S, Choi HK, Della-Torre E, Dicaire JF, et al. The 2019 American college of rheumatology/European league against rheumatism classification criteria for IgG4-related disease. Ann Rheum Dis. 2020;79:77-87.

4. Wu A, Andrew NH, McNab AA, Selva D. Bilateral IgG4-related ophthalmic disease: a strong indication for systemic imaging. Br J Ophthalmol. 2016;100:1409-11.

5. Hong JW, Kang S, Song MK, Ahn CJ, Sa HS. Clinicoserological factors associated with response to steroid treatment and recurrence in patients with IgG4-related ophthalmic disease. $\mathrm{Br} \mathrm{J}$ Ophthalmol. 2018;102:1591-5.

6. Wallace ZS, Mattoo H, Mahajan VS, Kulikova M, Lu L, Deshpande V, et al. Predictors of disease relapse in IgG4-related disease following rituximab. Rheumatology (Oxford). 2016;55:1000-8.

7. Peng Y, Li JQ, Zhang PP, Zhang X, Peng LY, Chen H, et al. Clinical outcomes and predictive relapse factors of IgG4-related disease following treatment: a long-term cohort study. J Intern Med. 2019;286:54252.

8. Mizushima I, Konishi M, Sanada H, Suzuki K, Takeji A, Zoshima T, et al. Serum IgG4 levels at diagnosis can predict unfavorable outcomes of untreated patients with IgG4-related disease. Sci Rep. $2021 ; 11: 13341$.

9. Carruthers MN, Stone JH, Deshpande V, Khosroshahi A. Development of an IgG4-RD responder index. Int J Rheumatol. 2012;2012:259408.

10. Matsubayashi H, Sawai H, Kimura H, Yamaguchi $Y$, Tanaka M, Kakushima N, et al. Characteristics of autoimmune pancreatitis based on serum IgG4 level. Dig Liver Dis. 2011;43:731-5.

11. Culver EL, Sadler R, Simpson D, Cargill T, Makuch M, Bateman AC, et al. Elevated serum IgG4 levels in diagnosis, treatment response, organ involvement, and relapse in a prospective IgG4-related disease UK cohort. Am J Gastroenterol. 2016;111:733-43.

12. Kamisawa T, Okamoto A, Funata N. Clinicopathological features of autoimmune pancreatitis in relation to elevation of serum IgG4. Pancreas. 2005;31:28-31.

13. Hourai R, Ozawa H, Sohmiya K, Hirose Y, Katsumata T, Daimon M, et al. IgG4-positive plasmacytic infiltration in aortic wall and aortic valve surgical samples and its relation to preoperative serum IgG4 
levels. Int Heart J. 2019;60:688-94.

14. Deshpande V, Zen Y, Chan JK, Yi EE, Sato Y, Yoshino T, et al. Consensus statement on the pathology of IgG4-related disease. Mod Pathol. 2012;25:1181-92.

15. Sogabe Y, Ohshima K, Azumi A, Takahira M, Kase S, Tsuji H, et al. Location and frequency of lesions in patients with IgG4-related ophthalmic diseases. Graefes Arch Clin Exp Ophthalmol. 2014;252:531-8.

16. Sato Y, Ohshima K, Ichimura K, Sato M, Yamadori I, Tanaka T, et al. Ocular adnexal IgG4-related disease has uniform clinicopathology. Pathol Int. 2008;58:465-70.

17. Woo YJ, Kim JW, Yoon JS. Clinical implications of serum IgG4 levels in patients with IgG4-related ophthalmic disease. Br J Ophthalmol. 2017;101:256-60.

18. Ebbo M, Patient M, Grados A, Groh M, Desblaches J, Hachulla E, et al. Ophthalmic manifestations in IgG4-related disease: clinical presentation and response to treatment in a French case-series. Medicine (Baltimore). 2017;96:e6205.

19. Dragan AD, Weller A, Lingam RK. Imaging of IgG4-related disease in the extracranial head and neck. Eur J Radiol. 2021;136:109560.

20. Song YS, Choung HK, Park SW, Kim JH, Khwarg SI, Jeon YK. Ocular adnexal IgG4-related disease: CT and MRI findings. Br J Ophthalmol. 2013;97:412-8.

21. Park J, Lee MJ, Kim N, Kim JE, Park SW, Choung HK, et al. Risk factors for extraophthalmic involvement and treatment outcomes in patients with IgG4-related ophthalmic disease. $\mathrm{Br} \mathrm{J}$ Ophthalmol. 2017;102:736-41.

22.Vinuesa CG, Tangye SG, Moser B, Mackay CR. Follicular B helper T cells in antibody responses and autoimmunity. Nat Rev Immunol. 2005;5:853-65.

\section{Figures}



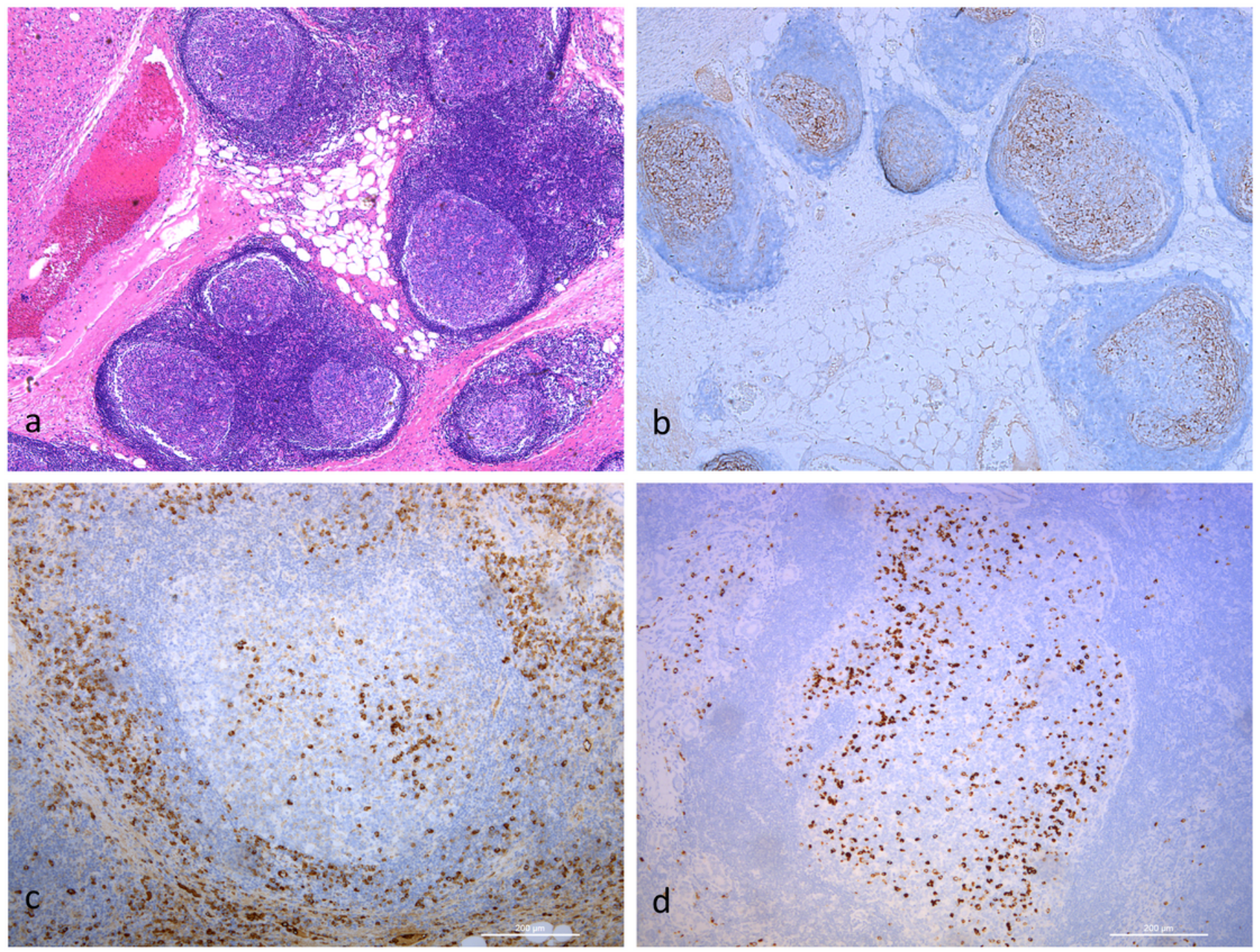

\section{Figure 1}

GCs and infiltrating IgG4+ plasma cells in lesions. (a, b) Lacrimal gland from patient no. 7 showed abundant GCs that were confirmed by CD21 immunostaining (hematoxylin and eosin, a, b: 50x). (c) A GC was surrounded by numerous IgG4-positive cells and fibrosis. Only sparse lgG4+ plasma cells were distributed within the GC (100x). (d) A majority of IgG4+ plasmacytes were confined to GCs (100x). 
a

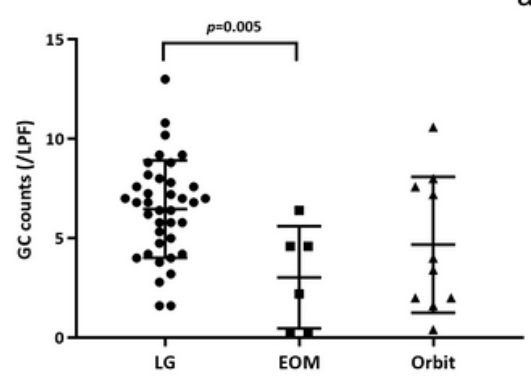

d

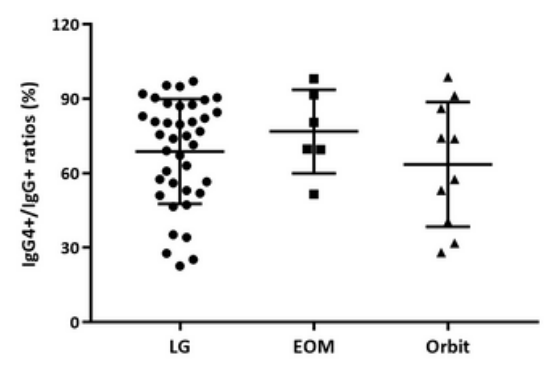

b

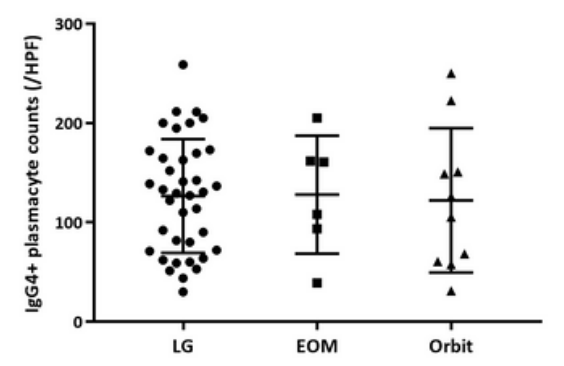

e



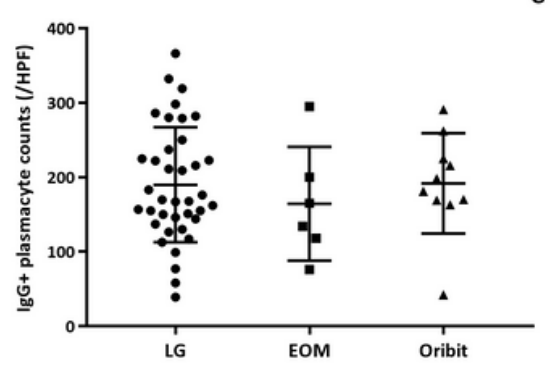

$C$

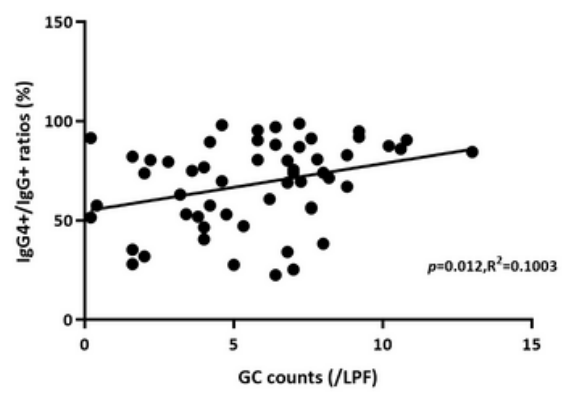

Figure 2

Quantity of pathological factors in lesions from different ophthalmic sites. (a) GC. (b, c, d) IgG4+ plasmacytes, IgG+ plasmacytes and their ratio. Correlation between the counts of GCs and infiltrating IgG4+ plasmacytes. (e) GC and IgG4+ plasmacyte. (f) GC and IgG4+/lgG+ ratio.

a


b

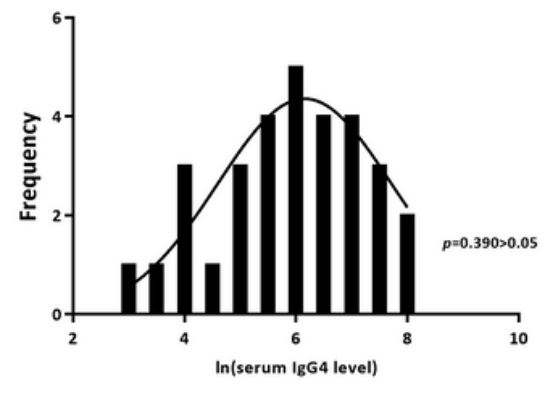

e

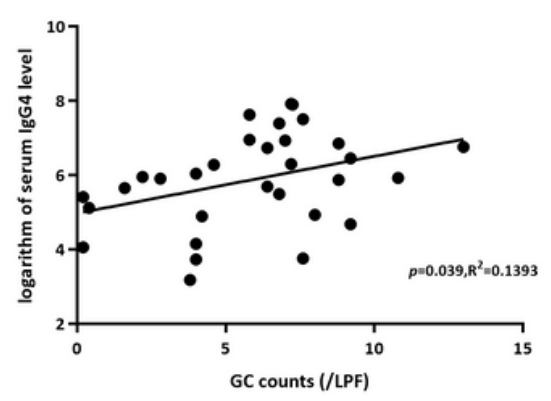

C
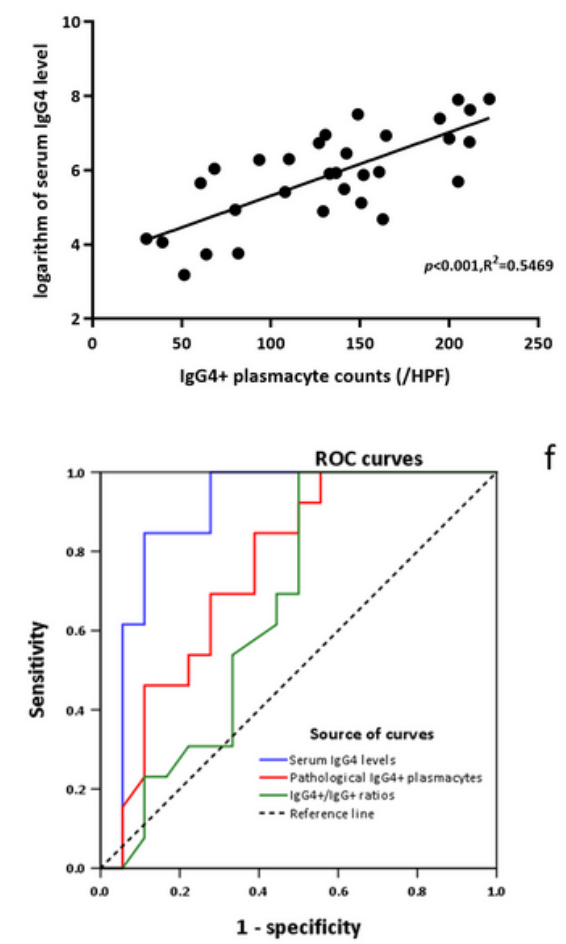


\section{Figure 3}

The distribution of the serum IgG4 level and its logarithm in patients with IgG4ROD. (a) Serum IgG4 level displayed a highly skewed distribution. (b) Logarithm of serum IgG4 level displayed a normal distribution. The correlation between the logarithm of the serum IgG4 level and pathological factors. (c) IgG4+ plasmacyte and In(serum IgG4 level). (d) IgG4+/lgG+ ratio and In(serum IgG4 level). (e) GC and In(serum IgG4 level). Receiver operating characteristic curve (ROC) analysis. (f) ROC curves to evaluate the predictive performance of serum IgG4 level, IgG4+ plasmacyte, and lgG4+/lgG+ ratio for systemic involvement.

a



b

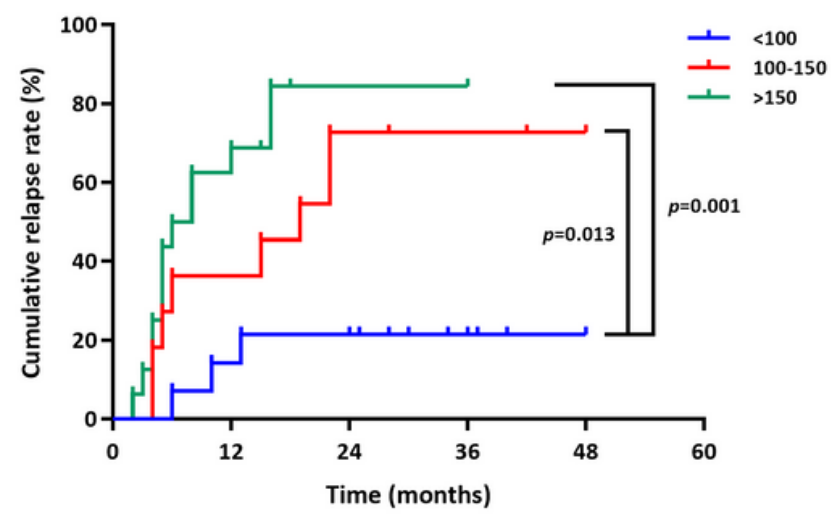

\section{Figure 4}

Forest plot of multivariate Cox regression analysis and curves of cumulative relapse rate for patients with IgG4ROD. (a) Forest plot of multivariate Cox regression analysis for IgG4-ROD relapse. Pathological IgG4+ plasmacyte count of $>150 / \mathrm{HPF}$ and elevated serum IgG4 level of $>500 \mathrm{mg} / \mathrm{dL}$ were risk factors associated with relapse after steroid treatment. (b) Kaplan-Meier curve of the cumulative relapse rate of IgG4ROD patients based on the grade of IgG4+ plasmacyte infiltration. The difference between curves of $100-150$ and $>150$ was not significant $(p=0.148>0.05)$. HR hazard ratio, $\mathrm{Cl}$ confidence interval, GC germinal center. ${ }^{*} p<0.05$. 